\title{
Use of Intuitionistic Fuzzy Numbers in Survey Sampling Analysis with Application in Electronic Data Interchange
}

\author{
Farah Rasheed $\left(\mathbb{D},{ }^{1}\right.$ Sajida Kousar $\left(\mathbb{D},{ }^{1}\right.$ Javid Shabbir $\left(\mathbb{D},{ }^{2}\right.$ Nasreen Kausar $\left(\mathbb{D},{ }^{3}\right.$ \\ Dragan Pamucar $\mathbb{C}^{4},{ }^{4}$ and Yaé Ulrich Gaba $\mathbb{1}^{5,6,7}$ \\ ${ }^{1}$ Department of Mathematics and Statistics, International Islamic University Islamabad, Islamabad, Pakistan \\ ${ }^{2}$ Department of Statistics, Quaid-i-Azam University, Islamabad, Pakistan \\ ${ }^{3}$ Department of Mathematics, Faculty of Arts and Science, Yildiz Technical University, Esenler, 34210 Istanbul, Turkey \\ ${ }^{4}$ Department of Logistics, University of Defence in Belgrade, Belgrade, Serbia \\ ${ }^{5}$ Quantum Leap Africa (QLA), AIMS Rwanda Center, Remera Sector KN 3, Kigali, Rwanda \\ ${ }^{6}$ Institut de Mathématiques et de Sciences Physiques (IMSP/UAC), Laboratoire de Topologie Fondamentale, \\ Computationnelle et Leurs Applications (Lab-ToFoCApp), BP 613, Porto-Novo, Benin \\ ${ }^{7}$ African Center for Advanced Studies, P.O. Box 4477, Yaounde, Cameroon
}

Correspondence should be addressed to Yaé Ulrich Gaba; yaeulrich.gaba@gmail.com

Received 17 August 2021; Revised 17 September 2021; Accepted 18 September 2021; Published 19 October 2021

Academic Editor: Borna Abramović

Copyright (C) 2021 Farah Rasheed et al. This is an open access article distributed under the Creative Commons Attribution License, which permits unrestricted use, distribution, and reproduction in any medium, provided the original work is properly cited.

The validity of survey sampling depends on how precise the data is. The validity reduces when questionnaire contains linguistic variables and respondent has to answer in zero or one. The ambiguity in the respondent's mind can be minimized by getting a response in an intuitionistic fuzzy sense. In this study, we proposed intuitionistic fuzzy aggregative investment benefit rate to select the best manufacturing facility location, where respondents expressed both membership function and nonmembership function for linguistic variables, with some hesitancy level, and give significant improvement over the fuzzy assessment method. The proposed methodology is also verified at different hesitancy levels and recommended to use a small hesitancy level. Real-life example of the use of electronic data interchange (EDI) in Pakistan's different fields, such as business, commerce, transport, airlines, warehousing, and logistic and delivery industry, is used to support the proposed methodology. Thus, an intuitionistic fuzzy rate reduces the cost, time, and effort of survey sampling instead of taking and analyzing responses from large samples.

\section{Introduction}

The traditional way of conducting research in social sciences, marketing, business, economics, health services, and psychological research is survey sampling. In these fields, information is collected from a sample of individuals through their responses to certain questions. Survey sampling research is categorized into quantitative (using a questionnaire with numerically rated items), qualitative (using a questionnaire with binary, open-ended, closed-ended, and rating scale questions) and mixed research procedures (both numerically rated and rating scale questions). In qualitative research, binary and rating scale questions are coded and then statistically analyzed, which leads to bias results as human thinking is full of ambiguity. Such types of questions are fuzzy in nature and cannot be expressed by binary and ordered numbers. Survey questions can be of various types, for example, multiple choice questions, rating scale questions, Likert scale questions, and matrix questions. Likert scale questions are widely used in social sciences, marketing business, and psychological research as these are perfect in measuring respondent's attitudes and behavior. For example, "there were enough toppings on my pizza," and the ratings might strongly agree, agree, neutral, disagree, and strongly disagree. These ratings are linguistic variables and the respondents are asked to pick just one answer; marked answer will get one numerical value while unmarked linguistic variables will be considered as zero. In multiple 
answer rating questions, respondents are asked to mark multiple answers. The representation of linguistic variables as a crisp variable does not represent the ambiguity of the respondent's mind. Thus, the linguistic variables are naturally vague. The problem of imprecise crisp response can be overcome by using an intuitionistic fuzzy (IF) response. In IF sense, respondents are requested to report responses in the form of the grade of membership and grade of nonmembership to linguistic variables at the given hesitancy level, where grade of membership and nonmembership are the values between zero and one, and their sum should be less than one. In 2020, Khani and Afkhami [1] conducted a sample survey using the interview method to analyze the issues which are related to the relationship between the language and the community. They examined the phonological changes in the Turkish dialect of Qazvin, according to the effects of social variables, such as age and gender.

In 1965, Zadeh [2] defined the grade of membership of a component belonging to a universal set as a value between zero and one, but the grade of nonmembership is not a complement of the grade of membership because of the hesitancy grade. Later, the fuzzy number was introduced Zadeh $[3,4]$ to represent the linguistic variables. Lin and Lee $[5,6]$ discussed survey sampling in fuzzy environment. Li et al. [7] used fuzzy sampling survey to distinguish the endogenous and exogenous factors influencing regional tourism and developed theory to explain the basic countermeasures for promoting regional tourism cooperation in fuzzy sense. Tavares and Betti [8] used fuzzy set approach as complements to measure multidimensional poverty within the context of the coronavirus pandemic. They used rank correlation analyzes and proposed indexes that can trace the trends in increasing infection and a higher mortality rate in vulnerable regions. Compared to headcount ratio results, the fuzzy measures have more precise outcomes and are better able to capture the evolution in mortality patterns.

In 1986, Atanassove [11] introduced the concept of intuitionistic fuzzy set(IFS), as an extension of fuzzy set theory to deal with imprecision. In 1994, Atanassove [9] proved its significance and importance in dealing with vagueness and described membership degree, nonmembership degree, and the degree of hesitation to each element of a set. Radhika and Parvathi [12] and Thangavelu et al. [13] described the stages of IFS as

(i) Intuitionistic fuzzification (converting crisp into membership and nonmembership function)

(ii) Application of operation rules of IFS

(iii) Intuitionistic defuzzification (converting membership and nonmembership to crisp)

In 2021, Isik and Kaya [14] combined the $\alpha$-cut technique of intuitionistic fuzzy set theory in acceptance sampling with the linguistic approach to allow defining with multiple $\alpha$ values for different product segments with the help of numerical examples. In 2020, Yuhana et al. [15] applied proposed rough and fuzzy sets to investigate the important factors that affect the value of the minimum passing level (MPL) of competency achievement. They predicted the category of the MPL using the combination of rough sets and fuzzy signatures method and evaluated its performance. They collected data from fifteen headmasters and sixty teachers of elementary schools participated. Based on the experiment with 203 objects' data, their proposed method gave $97 \%$ accuracy in the identification of important factors and prediction of MPL. Precup et al. [16] evolved fuzzy models (FMs) to develop an incremental online identification algorithm that characterize the nonlinear finger dynamics of the human hand for the myoelectric (ME)-based control of a prosthetic hand. They designed five simple Takagi-Sugeno proportional-integral (PI) controllers and showed that these fuzzy controllers are cost effective because of the simplicity of their structure. They proved with the simulation study that the best evolved FMs of the process have good performance in the control system with fuzzy controllers than the control system with linear ones. The fuzzy set theory is rapidly applied in all fields of statistics to deal with imprecise data and response; however, to the best of our knowledge, IF is not applied in survey sampling. This paper shows the significant contribution of IF in survey sampling at different hesitancy levels to find the aggregative investment benefit rate for the selection of the best facility location of multinational enterprises and compares the results with the fuzzy assessment method. A standard electronic format that puts back paperbased documents such as purchase orders or invoices is known as electronic data interchange (EDI). By self-operating paperbased transactions, organizations can save time and remove costly errors caused by manual processing. A survey report, in 2015, contains short analysis of the EDI implementation trends, based on data between 2005 and 2016, as well as estimation for year 2017 in various industries. In this report, Pakistan was not included in the list of countries using EDI standards in transport, warehousing, logistics, and delivery. This study also discusses the intuitionistic fuzzy assessment with the hesitancy level showing ambiguity in experts' mind, on the implementation of EDI.

\section{Preliminaries}

In 1965, Zadeh defined the fuzzy set as follows.

Definition 1. For a nonempty $x$, a pair $\widetilde{A}=(x, \mu \sim)$, where $\widetilde{A}$ to $[0,1]$ is called a fuzzy set; the function $\mu_{\widetilde{A}}$ is called the membership function of the set $\widetilde{A}$. More precisely, it is defined as

$$
\widetilde{A}=\left\{\left(x, \mu_{\tilde{A}}(X)\right) ; x \in X \text { and } \mu_{\tilde{A}}(x) \in[0,1]\right\} .
$$

A fuzzy set is called normalized if $\mu_{A}(X)=1$, for some $x \in X$. For $\alpha \in[0,1]$, the set,

$$
\widetilde{A}_{\alpha}=\left\{x \in X: \mu_{A} \geq \alpha\right\}
$$

is called $\alpha$ - level set of $\widetilde{A}$. For example, if $X$ is the set of real number and we define $\mu_{\tilde{A}}: X \longrightarrow[0,1]$ as $\mu_{\tilde{A}}(x)=\mid \sin$ $x \mid \forall x \in X$, then $\widetilde{A}=\left(x, \mu_{A}\right)$ is a fuzzy set, and for $\alpha=0.5, \widetilde{A}_{\alpha}=$ $\{x \in X:|\operatorname{Sin} x| \geq 0.5\}$ is an $\alpha-$ level set of $\widetilde{A}$. The fuzzy number is a normalized fuzzy set $\widetilde{A}$ of the set of real numbers if, for any $\lambda \in[0,1]$ and $(a, b) \epsilon \widetilde{A},(\lambda a+(1-\lambda) b) \epsilon \widetilde{A}$. 
Definition 2. triangular fuzzy number was introduced with arithmetic properties to get better conclusion in decisionmaking and real-life uncertain situations than fuzzy logic. $\widetilde{A}=\left(\widetilde{a}_{1}, \widetilde{a}_{2}, \widetilde{a}_{3}\right)$ is defined to be a triangular fuzzy number if its membership of $\mu_{\tilde{A}}: R \longrightarrow[0,1]$ is equal to

$$
\mu_{A}(x)= \begin{cases}\frac{x-\tilde{a}_{1}}{\tilde{a}_{2}-\tilde{a}_{1}}, & \text { if } x \in\left[\tilde{a}_{2}, \tilde{a}_{1}\right], \\ \frac{\tilde{a}_{3}-x}{\widetilde{a}_{3}-\tilde{a}_{2}}, & \text { if } x \in\left[\tilde{a}_{3}, \tilde{a}_{2}\right], \\ 0, & \text { otherwise, }\end{cases}
$$

where $\tilde{a}_{1} \leq \widetilde{a}_{2} \leq \widetilde{a}_{3}$.

Atanassov [10] modified the concept of standard fuzzy set by introducing the notion of nonmembership function $v_{A}: X \longrightarrow[0,1]$. He termed his finding as intuitionistic fuzzy set(IFS). So, by an IFS, we mean the triplet $\left(x, \mu_{\widetilde{A}}, \nu_{\widetilde{A}}\right)$ and the relation between the elements of $x$, and their images under $\mu_{\tilde{A}}$ and $\nu_{\tilde{A}}$ can be expressed as

$$
\widetilde{A}=\left\{\left\langle x, \mu_{\tilde{A}}(x), \nu_{\widetilde{A}}(x)\right\rangle: x \in X, \mu_{\widetilde{A}}(x), \nu_{\widetilde{A}}(x) \in[0,1]\right\} .
$$

The idea of nonmembership function is not identical to the standard concept of probability of occurrence $P$ and nonoccurrence $(1-P)$ of a certain event in classical probability theory. That is, in IFS, $\mu_{\mathcal{A}}(x)+\nu_{\widetilde{A}}(x) \leq 1$ which means that there must be some degree of hesitancy in giving response to a particular event. For instance, $X=N$, $\mu_{\tilde{A}}: N \longrightarrow[0,1]$ as $\mu_{\tilde{A}}(n)=1 / n+1$, and $\nu_{\widetilde{A}}(n)=1 / n+2$; then, $\quad \mu_{\mathcal{A}}(n)+\nu_{\widetilde{A}}(n)=1 / n+1+1 / n+2=n+2+n+1 /$ $(n+1)(n+2)=2 n+3 /(n+1)(n+2)$. For $n=1,5 /(2)$ (3) $<1$, and for $n=2,(7 /(3)(4))<1$.

Atanassov $[9,10]$ defined the algebraic operations on IFS as follows:

$$
\begin{aligned}
\widetilde{A}+\widetilde{B} & =\left\{\left\langle x, \mu_{\widetilde{A}}(x)+\mu_{\widetilde{B}}(x)-\mu_{\widetilde{A}}(x) * \mu_{\widetilde{B}}, \nu_{\widetilde{A}}(x) \nu_{\widetilde{B}}(x)\right\rangle: x \in X\right\}, \\
\widetilde{A} \cdot \widetilde{B} & =\left\{\left\langle\mu_{\widetilde{A}}(x) \mu_{\widetilde{B}}(x), \nu_{\widetilde{A}}(x)+\nu_{\widetilde{B}}(x)-\nu_{\widetilde{A}}(x) \nu_{\widetilde{B}}(x)\right\rangle: x \in X\right\}, \\
n \widetilde{A} & =\left\{\left\langle x, 1-\left[1-\mu_{\widetilde{A}}(x)\right]^{n},\left[\nu_{\widetilde{A}}(x)\right]^{n}\right\rangle: x \in X\right\}, \\
\widetilde{A}^{n} & =\left\{\left\langle x,\left[\mu_{\widetilde{A}}(x)\right]^{n}, 1-\left[1-\nu_{\widetilde{A}}(x)\right]^{n}\right\rangle: x \in X, n>0\right\} .
\end{aligned}
$$

Definition 3. A triangular intuitionistic fuzzy number (TIFN) is $\widetilde{A}^{*}=\left\{\left(a_{1}, a_{2}, a_{3}\right),\left(a_{1}^{*}, a_{2}^{*}, a_{3}^{*}\right)\right\}$ with $\left(a_{1}^{*}, a_{2}, a_{3}^{*}\right) \leq$ $\left(a_{1}, a_{2}, a_{3}\right)^{c}$, i.e., either $a_{1}^{*} \geq a_{2}, a_{2}^{*} \geq a_{3}$ or $a_{2}^{*} \leq a_{1}, a_{3}^{*} \leq a_{2}$ are membership and nonmembership fuzzy numbers of $\widetilde{A}^{*}$.

Definition 4. Zhang and Liu [17] described the operations on the intuitionistic fuzzy number as follows.

Let $\widetilde{A}^{*}=\left\{\left(a_{1}, a_{2}, a_{3}\right)\left(a_{1}^{*}, a_{2}^{*}, a_{3}^{*}\right)\right\}$ and $\widetilde{B}^{*}=\left\{\left(b_{1}, b_{2}, b_{3}\right)\right.$ $\left.\left(b_{1}^{*}, b_{2}^{*}, b_{3}^{*}\right)\right\}$ be two triangular intuitionistic fuzzy numbers. The arithmetic operation on $\widetilde{A}^{*}$ and $\widetilde{B}^{*}$ are given below:

\section{Addition:}

$$
\begin{aligned}
& \cdot\left(a_{1}, a_{2}, a_{3}\right)\left(a_{1}^{*}, a_{2}^{*}, a_{3}^{*}\right)+\left(b_{1}, b_{2}, b_{3}\right)\left(b_{1}^{*}, b_{2}^{*}, b_{3}^{*}\right) \\
= & \left(a_{1}+b_{1}, a_{2}+b_{2}, a_{3}+b_{3} ; a_{1}^{*}+b_{1}^{*}, a_{2}^{*}+b_{2}^{*}, a_{3}^{*}+b_{3}^{*}\right),
\end{aligned}
$$

Subtraction:

$$
\begin{aligned}
& \cdot\left(a_{1}, a_{2}, a_{3}\right)\left(a_{1}^{*}, a_{2}^{*}, a_{3}^{*}\right)+\left(b_{1}, b_{2}, b_{3}\right)\left(b_{1}^{*}, b_{2}^{*}, b_{3}^{*}\right) \\
= & \left(a_{1}-b_{3}, a_{2}-b 2, a_{3}-b_{1}, a_{1}^{*}-b_{3}^{*}, a_{2}^{*}-b_{2}^{*}, a_{3}^{*}-b_{3}^{*}\right),
\end{aligned}
$$

\section{Multiplication:}

$$
\begin{aligned}
& \cdot\left(a_{1}, a_{2}, a_{3}\right)\left(a_{1}^{*}, a_{2}^{*}, a_{3}^{*}\right) *\left(b_{1}, b_{2}, b_{3}\right)\left(b_{1}^{*}, b_{2}^{*}, b_{3}^{*}\right) \\
= & \left(a_{1} b_{1}, a_{2} b_{2}, a_{3} b_{3}\right)\left(a_{1}^{*} b_{1}^{*}, a_{2}^{*} b_{2}^{*}, a_{3}^{*} b_{3}^{*}\right),
\end{aligned}
$$

\section{Scalar Multiplication:}

$$
\begin{aligned}
& \cdot k\left(a_{1}, a_{2}, a_{3}\right)\left(a_{1}^{*}, a_{2}^{*}, a_{3}^{*}\right) \\
= & \left(k a_{1}, k a_{2}, k a_{3}\right)\left(k a_{1}^{*}, k a_{2}^{*}, k a_{3}^{*}\right) .
\end{aligned}
$$

Definition 5. Thangavelu et al. [13] defined average function

$$
D\left(A^{*}\right)=\frac{\left(a_{1}+4 a_{2}+a_{3}\right)+\left(a_{1}^{*}+4 a_{2}^{*}+a_{3}^{*}\right)}{12}
$$

to defuzzify a triangular intuitionistic fuzzy number.

\section{Questionnaire in Sample Surveys}

At the beginning of twenty first century, the decisionmaking problem about the selection of manufacturing facility locations is a demanding task. The conventional factors used in facility site selection were manufacturing site wages, infrastructure, education, workforce development, proximity to market, etc. Many new factors such as political stability, social harmony, trade regulations, and nature of governments' environmental consideration are added as pivotal factors in the decision-making problem of facility site selection. The achievement of a site selection scheme can be directly imputed to projects that begin with a checklist of matters and factors, appropriately weighted and estimated for the client's particular demands. Lin and Lee [6] proposed a new fuzzy assessment model to assist in finding the best facility site of multinational enterprises (MNEs) based on various investment environments. They proposed the fuzzy assessment model which is not only easier but also closer to evaluator real thinking and more useful than the ones presented before. For such type of surveys, questionnaire may include main survey items and subitems may exist under each main item. We define them as 
Main item: $C_{1}, C_{2}, \ldots, C_{n}$

with weights: $c_{1}, c_{2}, \ldots, c_{n}$, respectively,

subject to: $0 \leq c_{i} \leq 1, i=1,2, \ldots, n$, and $\sum_{i=1}^{n} a_{i}=1$,

subitem: $C_{i 1}, C_{i 2}, \ldots, C_{i m_{i}}$ under main items, $j=1,2, \ldots, m_{i}$,

with weights: $c_{i 1}, c_{i 2}, \ldots, c_{i m_{i}}$, respectively,

subject to: $0 \leq c_{i j} \leq 1, j=1,2, \ldots, m_{i}$, and $\sum_{j}^{m_{i}} c_{i j}=1$.

Let $A_{u}$, for $u=1,2, \ldots, k$, be the $k$ different linguistic variables such as very low, low, medium, high, and very high. These linguistic variables are rating scale question which requires a person to rate it along a well-defined, evenly spaced continuum. Rating scales are often used to measure the direction and intensity of attitudes of interviewee or respondents. The above questionnaire can be described as in Table 1 . The rating scale questions can be answered in two ways, single rating choice answer and multiple-rating choice answer. In single rating choice answer, the respondent/interviewee is requested to say yes to only one option and zero to other options. And, in multiple-rating answer questions, respondents can be requested to say yes for more than one option.

3.1. The Crisp Mode. Suppose we have a sample of $t$ respondents drawn from the population; each respondent rate more than 1 option among $A_{u}$, for $u=1,2, \ldots, k$, respectively, denotes the selected option by 1 , otherwise denoted by zero in the crisp case. The sample data are shown in Table 2.

Single rating choice answer:

$$
\begin{aligned}
\mu_{i j l u} & =0 \text { or } 1, \\
0 & \leq \sum_{u=1}^{k} \mu_{i j l u} \leq 1, \quad \text { for } l=1,2, \ldots, t \text { and } u=1,2, \ldots, k .
\end{aligned}
$$

Multiple-rating choice answer:

$$
\begin{aligned}
\mu_{i j l u} & =0 \text { or } 1, \\
1 & \leq \sum_{u=1}^{k} \mu_{i j l u} \leq k, \quad \text { for } l=1,2, \ldots, t \text { and } u=1,2, \ldots, k .
\end{aligned}
$$

3.2. The Intuitionistic Fuzzy Mode. The linguistic variables express the attitude of respondents, but the answer in yes coded as 1 and not coded as 0 cannot express the ambiguity in the mind of interviewee. If we request to interviewee to use intuitionistic fuzzy sense to give his response, the sample data can be obtained in the form of Table 3. In Table 3, $\mu_{i j l u} \epsilon[0,1]$ and $v_{i j l u} \epsilon[0,1]$ such that $\mu_{i j l u}+v_{i j l u}+\epsilon=1$, where $0 \leq \epsilon<1$ and satisfies the following conditions.
Single rating choice answer:

$$
\begin{aligned}
& 0<\sum_{u=1}^{k} \mu_{i j l u} \leq 1, \\
& 0<\sum_{u=1}^{k} v_{i j l u} \leq 1 .
\end{aligned}
$$

Multiple rating choice answer:

$$
\begin{aligned}
& 1<\sum_{u=1}^{k} \mu_{i j l u} \leq k, \\
& 1<\sum_{u=1}^{k} v_{i j l u} \leq k .
\end{aligned}
$$

The average of the membership function and nonmembership function of $t$ samples according to the above definitions, for each $A_{u}$, is denoted by $M_{i j l u}$ and $N_{i j l u}$, respectively:

$$
\begin{aligned}
\mu_{i j u}^{*} & =\sum_{l=1}^{t} \mu_{i j l u}-\prod_{l=1}^{t} \mu_{i j l u}, \\
v_{i j u}^{*} & =\prod_{l=1}^{t} \mu_{i j l u}, \\
M_{i j u} & =1-\left[1-\mu_{i j l u}^{*}\right]^{1 / t}, \\
N_{i j u} & =\left[v_{i j l u}^{*}\right]^{1 / t} .
\end{aligned}
$$

3.3. Weights'Selection. Every respondent/interviewee has to assign weights to each main item $C_{i}$ and subitem $C_{i j}$ according to relative importance. The traditional way is to assign ranks to each item. However, the crisp weight cannot express the hesitancy of the interviewee. So, we use the intuitionistic fuzzy number to express the grading of items. The range of grades is 0 to 10 , and we express the grades into triangular intuitionistic fuzzy numbers, as listed in Table 4. The membership and nonmembership of grades are

$$
\left\langle\mu_{A}(x), \nu_{A}(x)\right\rangle= \begin{cases}(0,1-\epsilon), & \text { if } x \leq 0, \\ \left\langle\left(\frac{x-0}{5-0}\right)-\epsilon, 1-\left(\frac{x-0}{5-0}\right)\right\rangle, & \text { if } 0<x \leq 5, \\ \left\langle\left(\frac{10-x}{10-5}\right)-\epsilon, 1-\left(\frac{10-x}{10-5}\right)\right\rangle, & \text { if } 5<x \leq 10, \\ (0,1-\epsilon), & \text { if } x \geq 10 .\end{cases}
$$

Suppose there are $t$ interviewees which assign weights $w_{i j l} \in\left[W_{0}, W_{1}, \ldots W_{10}\right], l=1,2, \ldots, t$, to each subitem $C_{i j l}$. Let for the $l^{\text {th }}$ expert $w_{i j l}=\left\{\left(a_{1 l}, a_{2 l}, a_{3 l}\right),\left(a_{1 l}^{*}, a_{2 l}^{*}, a_{3 l}^{*}\right)\right\}$. The average weight of $t$ experts is 
TABLE 1: Proposed questionnaire for assessing the aggregative benefit of facility site selection.

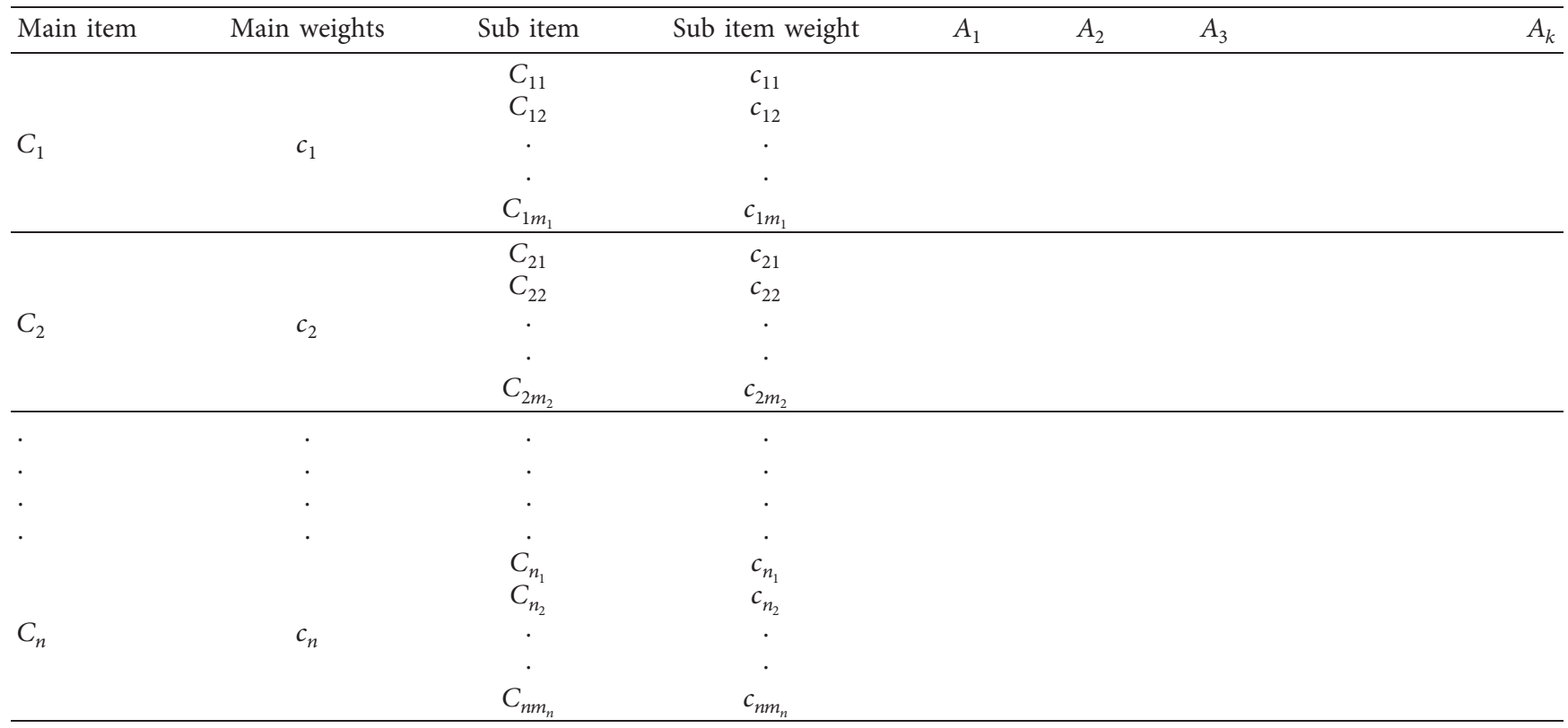

TABle 2: The sample data in the crisp case with multiple-rating choice answer.

\begin{tabular}{lcccc}
\hline Samples & $A_{1}$ & $A_{2}$ & $A_{3}$ & $A_{k}$ \\
\hline$S_{1}$ & $\mu_{i j 11}$ & $\mu_{i j 12}$ & $\mu_{i j 13}$ & $\mu_{i j 1 k}$ \\
$S_{2}$ & $\mu_{i j 21}$ & $\mu_{i j 22}$ & $\mu_{i j 23}$ & $\mu_{i j 2 k}$ \\
$\cdot$ & $\cdot$ & $\cdot$ & $\cdot$ & $\cdot$ \\
$\cdot$ & $\cdot$ & $\cdot$ & $\cdot$ & $\cdot$ \\
$\cdot$ & $\cdot$ & $\cdot$ & $\cdot$ & $\cdot$ \\
$S_{t}$ & $\mu_{i j t 1}$ & $\mu_{i j t 2}$ & $\mu_{i j t 3}$ & $\mu_{i j t k}$ \\
\hline
\end{tabular}

TABLE 3: The sample data in intuitionistic fuzzy case with multiple-rating choice answer.

\begin{tabular}{lcccc}
\hline Samples & $A_{1}$ & $A_{2}$ & $A_{3}$ & $A_{k}$ \\
\hline$S_{1}$ & $\left(\mu_{i j 11}, v_{i j 11}\right)$ & $\left(\mu_{i j 12}, v_{i j 12}\right)$ & $\left(\mu_{i j 13}, v_{i j 13}\right)$ & $\left(\mu_{i j 1 k}, v_{i j 1 k}\right)$ \\
$S_{2}$ & $\left(\mu_{i j 21}, v_{i j 21}\right)$ & $\left(\mu_{i j 22}, v_{i j 22}\right)$ & $\left(\mu_{i j 23}, v_{i j 23}\right)$ & $\left(\mu_{i j 2 k}, v_{i j 2 k}\right)$ \\
$\cdot$ & $\cdot$ & $\cdot$ & $\cdot$ & $\cdot$ \\
$\cdot$ & $\cdot$ & $\cdot$ & $\cdot$ & $\cdot$ \\
$\cdot$ & $\cdot$ & $\cdot$ & $\cdot$ & $\cdot$ \\
$S_{t}$ & $\left(\mu_{i j t 1}, v_{i j t 1}\right)$ & $\left(\mu_{i j t 2}, v_{i j t 2}\right)$ & $\left(\mu_{i j t 3}, v_{i j t 3}\right)$ & $\cdot$ \\
Average & $\left(M_{i j 1}, N_{i j 1}\right)$ & $\left(M_{i j 2}, N_{i j 2}\right)$ & $\left(M_{i j 3}, N_{i j 3}\right)$ & $\left(M_{i j k k}, v_{i j t k}\right)$ \\
\hline
\end{tabular}

$$
\begin{aligned}
w_{i j} & =\frac{1}{t} \otimes\left(\widetilde{A}_{1}^{*} \oplus \widetilde{A}_{2}^{*} \oplus \cdots \oplus \widetilde{A}_{t}^{*}\right) \\
& =\left\{\left(\frac{1}{t} \sum_{l=1}^{t} a_{1 l}, \frac{1}{t} \sum_{l=1}^{t} a_{2 l}, \frac{1}{t} \sum_{l=1}^{t} a_{3 l}\right),\left(\frac{1}{t} \sum_{l=1}^{t} a_{1 l}^{*}, \frac{1}{t} \sum_{l=1}^{t} a_{2 l}^{*}, \frac{1}{t} \sum_{l=1}^{t} a_{3 l}^{*}\right)\right\}
\end{aligned}
$$

and represented by $w_{i j}=\left\{\left(\bar{a}_{1}, \bar{a}_{2}, \bar{a}_{3}\right),\left(\bar{a}_{1}^{*}, \bar{a}_{2}^{*}, \bar{a}_{3}^{*}\right)\right\}$.

Then, defuzzified by equation $(7)$, we have

$$
D_{i j}=D\left(w_{i j}\right)=\frac{\left(\bar{a}_{1}+4 \bar{a}_{2}+\bar{a}_{3}\right)+\left(\bar{a}_{1}^{*}+4 \bar{a}_{2}^{*}+\bar{a}_{3}^{*}\right)}{12} .
$$


TABLE 4: Intuitionistic triangular fuzzy numbers for grades of items.

\begin{tabular}{lc}
\hline Grade & ITFN \\
\hline 0 & $W_{0}=([0,0,1],[2,3,4])$ \\
1 & $W_{1}=([0,1,2],[3,4,5])$ \\
2 & $W_{2}=([1,2,3],[4,5,6])$ \\
3 & $W_{3}=([2,3,4],[5,6,7])$ \\
4 & $W_{4}=([3,4,5],[6,7,8])$ \\
5 & $W_{5}=([4,5,6],[7,8,9])$ \\
6 & $W_{6}=([5,6,7],[8,9,10])$ \\
7 & $W_{7}=([6,7,8],[0,2,4])$ \\
8 & $W_{8}=([7,8,9],[1,3,5])$ \\
9 & $W_{9}=([8,9,10],[4,5,6])$ \\
10 & $W_{10}=([9,10,10],[7,8,9])$ \\
\hline
\end{tabular}

Let

$$
c_{i j}=\frac{D_{i j}}{\sum_{j=1}^{m_{i}} D_{i j}},
$$

where weights of the subitems $C_{i 1}, C_{i 2}, \ldots, C_{i m_{i}}$ are $c_{i 1}, c_{i 2}, \ldots, c_{i m_{i}}$.

Similarly, for $t$ interviewees, we assign weights $w_{i l} \in\left[W_{0}, W_{1}, \ldots, W_{10}\right]$, where $l=1,2, \ldots, t$ to each main item $C_{i l}$. Let for the $l^{\text {th }}$ expert $w_{i l}=\left\{\left(b_{1 l}, b_{2 l}, b_{3 l}\right)\right.$, $\left.\left(b_{1 l}^{*}, b_{2 l}^{*}, b_{3 l}^{*}\right)\right\}$.

The average weight of $t$ experts is

$$
\begin{aligned}
w_{i}= & \frac{1}{t} \otimes\left(\overline{L_{1}} \oplus \overline{L_{2}} \oplus \cdots \oplus \overline{L_{t}}\right) \\
= & \left\{\left(\frac{1}{t} \sum_{l=1}^{t} b_{1 l}, \frac{1}{t} \sum_{l=1}^{t} b_{2 l}, \frac{1}{t} \sum_{l=1}^{t} b_{3 l}\right),\right. \\
& \left.\cdot\left(\frac{1}{t} \sum_{l=1}^{t} b_{1 l}^{*}, \frac{1}{t} \sum_{l=1}^{t} b_{2 l}^{*}, \frac{1}{t} \sum_{l=1}^{t} b_{3 l}^{*}\right)\right\}, \\
w_{i}= & \left\{\left(\bar{b}_{1}, \bar{b}_{2}, \bar{b}_{3}\right),\left(\bar{b}_{1}^{*}, \bar{b}_{2}^{*}, \bar{b}_{3}^{*}\right)\right\} .
\end{aligned}
$$

Then, it is defuzzified as

$$
E_{i j}=E\left(w_{i}\right)=\frac{\left(\bar{b}_{1}+4 \bar{b}_{2}+\bar{b}_{3}\right)+\left(\bar{b}_{1}^{*}+4 \bar{b}_{2}^{*}+\bar{b}_{3}^{*}\right)}{12} .
$$

Let

$$
c_{i}=\frac{E_{i j}}{\sum_{j=1}^{n} E_{i j}},
$$

where weights of the main items $C_{1}, C_{2}, \ldots, C_{n}$ are $c_{i}, c_{i}, \ldots, c_{n}$.

\section{The Intuitionistic Aggregative Assessment for Survey Sampling}

Let $A=A_{1}, A_{2}, \ldots, A_{k}$ be the set of linguistic variables for each subitem. Using intuitionistic triangular fuzzy number and getting the rating (both single- and multiple-rating choice answer) in terms of the intuitionistic fuzzy mode, the results of survey sampling can be analyzed as follows:

Step 1: averaging assessment results of all respondents, an IF (intuitionistic fuzzy) evaluation matrix is as follows:

$$
R=\left[\begin{array}{cccc}
g_{11}\left(R_{1}\right) & g_{11}\left(R_{2}\right) & \ldots & g_{11}\left(R_{k}\right) \\
g_{12}\left(R_{1}\right) & g_{12}\left(R_{2}\right) & \ldots & g_{12}\left(R_{k}\right) \\
\cdot & \cdot & \ldots & \cdot \\
\cdot & \cdot & \ldots & \cdot \\
\cdot & \cdot & \ldots & \cdot \\
g_{n m_{n}}\left(R_{1}\right) & g_{n m_{n}}\left(R_{2}\right) & \ldots & g_{n m_{n}}\left(R_{k}\right)
\end{array}\right] \text {, }
$$

where $g_{i j}\left(R_{u}\right)=\left\{\left\langle R_{u}: \mu_{i j u}, v_{i j u}\right\rangle \mid i=1,2,3, \ldots, n ; j=\right.$ $\left.1,2, \ldots, m_{i}\right\}$.

Step 2: the normalize weighted vector for subitems is $c_{i j}=\left(c_{i 1}, c_{i 2}, \ldots, c_{i m_{i}}\right)$, and for the main item $C_{i}$, the first stage aggregative assessment is as

$$
\begin{aligned}
& R^{*}=\left(c_{i 1}, c_{i 2}, \ldots, c_{i m_{i}}\right) \\
& \times\left[\begin{array}{cccc}
g_{11}\left(R_{1}\right) & g_{11}\left(R_{2}\right) & \ldots & g_{11}\left(R_{k}\right) \\
g_{12}\left(R_{1}\right) & g_{12}\left(R_{2}\right) & \ldots & g_{12}\left(R_{k}\right) \\
\cdot & \cdot & \ldots & \cdot \\
\cdot & \cdot & \ldots & \cdot \\
\cdot & \cdot & \ldots & \cdot \\
g_{n m_{n}}\left(R_{1}\right) & g_{n m_{n}}\left(R_{2}\right) & \ldots & g_{n m_{n}}\left(R_{k}\right)
\end{array}\right] \\
& R^{*}=\left[\begin{array}{cccc}
f_{1}\left(R_{1}\right) & f_{1}\left(R_{2}\right) & \ldots & f_{1}\left(R_{k}\right) \\
f_{2}\left(R_{1}\right) & f_{2}\left(R_{2}\right) & \ldots & f_{2}\left(R_{k}\right) \\
\cdot & \cdot & \ldots & \cdot \\
\cdot & \cdot & \ldots & \cdot \\
\cdot & \cdot & \ldots & \cdot \\
f_{n}\left(R_{1}\right) & f_{n}\left(R_{2}\right) & \ldots & f_{n}\left(R_{k}\right)
\end{array}\right],
\end{aligned}
$$

where $\quad f_{i}\left(R_{u}\right)=\left\{\left\langle R_{u}, \mu_{i u}^{*}, v_{i u}^{*}\right\rangle \mid i=1,2,3, \ldots, n ; u=1,2\right.$, $\ldots, k\}$ and for $i^{\text {th }}$ main item $\mu_{i u}^{*}=\operatorname{Min}\left\{\sum_{j=1}^{m_{i}} c_{i j} \mu_{i j u}, 1\right\}$ and $v_{i u}^{*}=\operatorname{Min}\left\{\sum_{j=1}^{m_{i}} c_{i j} v_{i j u}, 1\right\}$. This is called the first stage intuitionistic fuzzy assessment matrix $R^{*}$.

Step 3: the normalized weight vector for main items is $c_{i}=\left(c_{1}, c_{2}, \ldots, c_{n}\right)$. The second stage intuitionistic fuzzy assessment is as follows: 


$$
\begin{aligned}
& \left(c_{1}, c_{2}, \ldots c_{n}\right) \times\left[\begin{array}{cccc}
f_{1}\left(R_{1}\right) & f_{1}\left(R_{2}\right) & \ldots & f_{1}\left(R_{k}\right) \\
f_{2}\left(R_{1}\right) & f_{2}\left(R_{2}\right) & \ldots & f_{2}\left(R_{k}\right) \\
\cdot & \cdot & \ldots & \cdot \\
\cdot & \cdot & \ldots & \cdot \\
\cdot & \cdot & \ldots & \cdot \\
f_{n}\left(R_{1}\right) & f_{n}\left(R_{2}\right) & \ldots & f_{n}\left(R_{k}\right)
\end{array}\right] \\
& =\left\{h\left(R_{1}\right), h\left(R_{2}\right), \ldots, h\left(R_{k}\right)\right\},
\end{aligned}
$$

where $\quad h\left(R_{u}\right)=\left\{\left\langle R_{u}, \tilde{\mu}_{u}, \tilde{v}_{u}\right\rangle \mid u=1,2, \ldots, k\right\} \quad$ and $\tilde{\mu}_{u}=\operatorname{Min}\left\{\sum_{i=1}^{n} c_{i} \mu_{i u}^{*}, 1\right\}, \tilde{v}_{u}=\operatorname{Min}\left\{\sum_{i=1}^{n} c_{i} v_{i u}^{*}, 1\right\} . \quad h\left(R_{u}\right)$ is second stage aggregative assessment with respect to the set of intuitionistic rating $A_{1}, A_{2}, \ldots A_{k}$ is

$$
I=\frac{\left(\tilde{\mu_{1}}, \tilde{\nu_{1}}\right)}{A_{1}}+\frac{\left(\tilde{\mu_{2}}, \tilde{\nu_{2}}\right)}{A_{2}}+\cdots+\frac{\left(\tilde{\mu_{k}}, \tilde{v_{k}}\right)}{A_{k}} \text {. }
$$

\section{Numerical Example}

In this section, we use example of Lin and Lee [6] to determine the aggregative benefit of investment for global facility site selection. In identifying the best facility site of multinational enterprises based on various environments and based on
TABLE 5: Intuitionistic triangular fuzzy numbers for rating of linguistic variables.

\begin{tabular}{lc}
\hline Rating & ITFN \\
\hline$A_{1}$ & $V_{1}=([0,0,1 / 6],[2 / 6,3 / 6,4 / 6])$ \\
$A_{2}$ & $V_{2}=([0,1 / 6,2 / 6],[3 / 6,4 / 6,5 / 6])$ \\
$A_{3}$ & $V_{3}=([1 / 6,2 / 6,3 / 6],[4 / 6,5 / 6,6 / 6])$ \\
$A_{4}$ & $V_{4}=([2 / 6,3 / 6,4 / 6],[5 / 6,6 / 6,6 / 6])$ \\
$A_{5}$ & $V_{5}=([3 / 6,4 / 6,5 / 6],[0 / 6,1 / 6,2 / 6])$ \\
$A_{6}$ & $V_{6}=([4 / 6,5 / 6,6 / 6],[1 / 6,2 / 6,3 / 6])$ \\
$A_{7}$ & $V_{7}=([5 / 6,6 / 6,6 / 6],[2 / 6,3 / 6,4 / 6])$ \\
\hline
\end{tabular}

identifying the critical factors that will affect the ongoing business, the linguistic variables used by Lin and Lee [6] to rate factors are $A_{1}, A_{2}, \ldots, A_{7}$, where $A_{1}=$ extralow, $A_{2}=$ extralow, $A_{3}=$ Low, $A_{4}=$ medium, $A_{5}=$ high, $A_{6}=$ very high, and $A_{7}=$ extra high to get aggregative benefit rate of facility site. These variables represented in triangular intuitionistic fuzzy numbers are shown in Table 5.

Assume two evaluators give weights and responses of factors effecting investment benefit with hesitancy error 0.05 in Tables 6 and 7, respectively.

Then, by equations (7), (17), and (20), the aggregative of responses of two evaluators is shown in Table 8.

Step 1: the intuitionistic fuzzy average response matrix $R$ is

$\left.\begin{array}{ccccc}(0.50000,0) & 0 & 0 & 0 & 0 \\ (0.5,0) & 0 & 0 & 0 & 0 \\ 0 & 0 & 0 & 0 & 0 \\ (0.6,0) & 0 & 0 & (0.5,0) & 0 \\ 0 & 0 & 0 & 0 & 0 \\ (0.45228,0) & (0.6127,0) & 0 & 0 & 0 \\ 0 & 0 & 0 & 0 & 0 \\ (0.5,0) & 0 & 0 & 0 & 0 \\ (0.45228,0.19365) & (0.55279,0) & 0 & 0 & 0 \\ 0 & 0 & 0 & 0 & 0\end{array}\right]$.

Step 2: the first stage intuitionistic fuzzy aggregative assessment for each main item $C_{i}$ is as

$$
R^{*}=\left[\begin{array}{ccccccr}
(0.214972,0) & (0.32447,0) & (0.5,0) & (0,0) & (0,0) & (0,0) & (0,0) \\
(0,0) & (0.33365,0.191631) & (0.268085,0) & (0,0) & (0,0) & (0,0) & (0,0) \\
(0.30375,0) & (0.33667,0) & (0.268085,0) & (0,0) & (0,0) & (0.223404,0) & (0,0) \\
(0.36878,0.074011) & (0.26596,0) & (0.26595,0) & (0,0) & (0,0) & (0,0) & (0,0) \\
(0,0) & (0.269889,0) & (0.23034, .098624) & (0.28153,0) & (0,0) & (0,0) & (0,0)
\end{array}\right] .
$$


TABLE 6: Responses of the first evaluator.

\begin{tabular}{|c|c|c|c|c|c|c|c|c|c|c|}
\hline Main item & Weight 1 & Subitem & Weight 2 & $A_{1}$ & $A_{2}$ & $A_{3}$ & $A_{4}$ & $A_{5}$ & $A_{6}$ & $A_{7}$ \\
\hline \multirow{2}{*}{$F_{1}$} & \multirow{2}{*}{$([2,3,4],[5,6,7])$} & $F_{11}$ & $([4,5,6],[7,8,9])$ & 0 & $(0.78,0.17)$ & 0 & 0 & 0 & 0 & 0 \\
\hline & & $F_{12}$ & $([0,1,2],[3,4,5])$ & 0 & 0 & $(0.75,0.2)$ & 0 & 0 & 0 & 0 \\
\hline \multirow{2}{*}{$F_{2}$} & \multirow{2}{*}{$([0,1,2],[3,4,5])$} & $F_{21}$ & $([5,6,7],[8,9,10])$ & 0 & $(0.55,0.4)$ & 0 & 0 & 0 & 0 & 0 \\
\hline & & $F_{22}$ & $(8,9,10],[4,5,6])$ & 0 & 0 & $(0.84,0.11)$ & 0 & 0 & 0 & 0 \\
\hline \multirow{2}{*}{$F_{3}$} & \multirow{2}{*}{$([4,5,6],[7,8,9])$} & $F_{31}$ & $([2,3,4],[5,6,7])$ & $(0.8,0.15)$ & 0 & 0 & 0 & 0 & 0 & 0 \\
\hline & & $F_{32}$ & $([6,7,8],[0,2,4])$ & 0 & 0 & $(0.7,0.25)$ & 0 & 0 & 0 & 0 \\
\hline \multirow{2}{*}{$F_{4}$} & \multirow{2}{*}{$([3,4,5],[6,7,8])$} & $F_{41}$ & $([1,2,3],[4,5,6])$ & $(0.7,0.25)$ & 0 & 0 & 0 & 0 & 0 & 0 \\
\hline & & $F_{42}$ & $([7,8,9],[1,3,5])$ & 0 & $(0.75,0.2)$ & 0 & 0 & 0 & 0 & 0 \\
\hline \multirow{2}{*}{$F_{5}$} & \multirow{2}{*}{$([1,2,3],[4,5,6])$} & $F_{51}$ & $([9,10,10],[7,8,9])$ & 0 & 0 & $(0.75,0.2)$ & 0 & 0 & 0 & 0 \\
\hline & & $F_{52}$ & $([3,4,5],[6,7,8])$ & 0 & $(0.55,0.4)$ & 0 & 0 & 0 & 0 & 0 \\
\hline
\end{tabular}

TABLE 7: Responses of the second evaluator.

\begin{tabular}{|c|c|c|c|c|c|c|c|c|c|c|}
\hline Main & Weight 1 & Subitem & Weight 2 & $A_{1}$ & $A_{2}$ & $A_{3}$ & $A_{4}$ & $A_{5}$ & $A_{6}$ & $A_{7}$ \\
\hline \multirow{2}{*}{$F_{1}$} & \multirow{2}{*}{$([3,4,5],[6,7,8])$} & $F_{11}$ & $([2,3,4],[5,6,7])$ & 0 & 0 & $(0.75,0.2)$ & 0 & 0 & 0 & 0 \\
\hline & & $F_{12}$ & $([6,7,8],[0,2,4])$ & $(0.8,0.15)$ & 0 & 0 & 0 & 0 & 0 & 0 \\
\hline \multirow{2}{*}{$F_{2}$} & \multirow{2}{*}{$([1,2,3],[4,5,6])$} & $F_{21}$ & $([3,4,5],[6,7,8])$ & 0 & $(0.65,0.3)$ & 0 & 0 & 0 & 0 & 0 \\
\hline & & $F_{22}$ & $([1,2,3],[4,5,6])$ & 0 & 0 & 0 & 0 & 0 & $(0.75,0.2)$ & 0 \\
\hline \multirow{2}{*}{$F_{3}$} & \multirow{2}{*}{$(0,1,2],[3,4,5]$} & $F_{31}$ & $([9,10,10],[7,8,9])$ & 0 & $(0.85, .1)$ & 0 & 0 & 0 & 0 & 0 \\
\hline & & $F_{32}$ & $([4,5,6],[7,8,9])$, & 0 & 0 & & $(0.85,0.1)$ & 0 & 0 & 0 \\
\hline \multirow{2}{*}{$F_{4}$} & \multirow{2}{*}{$([4,5,6],[7,8,9])$} & $F_{41}$ & $([5,6,7],[8,9,10])$ & $(0.85 .0 .1)$ & 0 & 0 & 0 & 0 & 0 & 0 \\
\hline & & $F_{42}$ & $([8,9,10],[4,5,6])$ & 0 & 0 & $(0.75,0.2)$ & 0 & 0 & 0 & 0 \\
\hline \multirow{2}{*}{$F_{5}$} & \multirow{2}{*}{$([2,3,4],[5,6,7])$} & $F_{51}$ & $([0,1,2],[3,4,5])$ & 0 & 0 & 0 & $(0.8,0.15)$ & 0 & 0 & 0 \\
\hline & & $F_{52}$ & $([7,8,9],[1,3,5])$ & 0 & $(0.55,0.4)$ & 0 & 0 & 0 & 0 & 0 \\
\hline
\end{tabular}

Step 3: the main intuitionistic fuzzy aggregative assessment membership and nonmembership rate for all linguistic variables $A_{1}, A_{2}, A_{3}, A_{4}, A_{5}, A_{6}, A_{7}$ are

(0.1893, 0.0197), (0.3170, 0.0386), (0.09473,0), (0,0), (0.0268, 0), (0, 0), (0, 0),

and the normalized IF membership and nonmembership rate are $(0.5971,0.0197),(1.0,0.0386),(0.2988,0),(0,0)$, $(0.0845,0),(0,0),(0,0)$.
And, this IF aggregative benefit for facility site selection is represented as

$$
\bar{I}=\frac{(0.1893,0.0197)}{A_{1}}+\frac{(0.3170,0.0386)}{A_{2}}+\frac{(0.09473,0)}{A_{3}}+\frac{(0,0)}{A_{4}}+\frac{(0.0268,0)}{A_{5}}+\frac{(0,0)}{A_{6}}+\frac{(0,0)}{A_{7}} .
$$

Defuzzifing the above, the IF aggregative investment membership and nonmembership rate, we have the IF investment benefit rate with 0.05 hesitancy rate is $(0.452331,0.021309)$. The fuzzy investment benefit rate calculated by Lin and Lee was 0.2039 . Thus, the IF proposed methodology gives better results than the fuzzy approach. With the hesitancy level, the decision-making become more reliable in uncertain situations. The intuitionistic fuzzy aggregative investment benefit with different hesitancy levels are shown in Table 9.
5.1. Application. Pakistan can boost its imports, exports, industry, and business by using electronic data interchange. The major cause of the stagnation of Pakistan's economy is the less use of EDI. To determine the intuitionistic fuzzy EDI implementation rate, the opinion is collected from experts rather than conducting a large opinion survey on the implementation of EDI. The hierarchical model on the implementation of EDI Pakistan is shown in Figure 1. The linguistic variables on the use of implementation of EDI in different fields are extra low, very low, low, middle, high, very high, and extra high. 


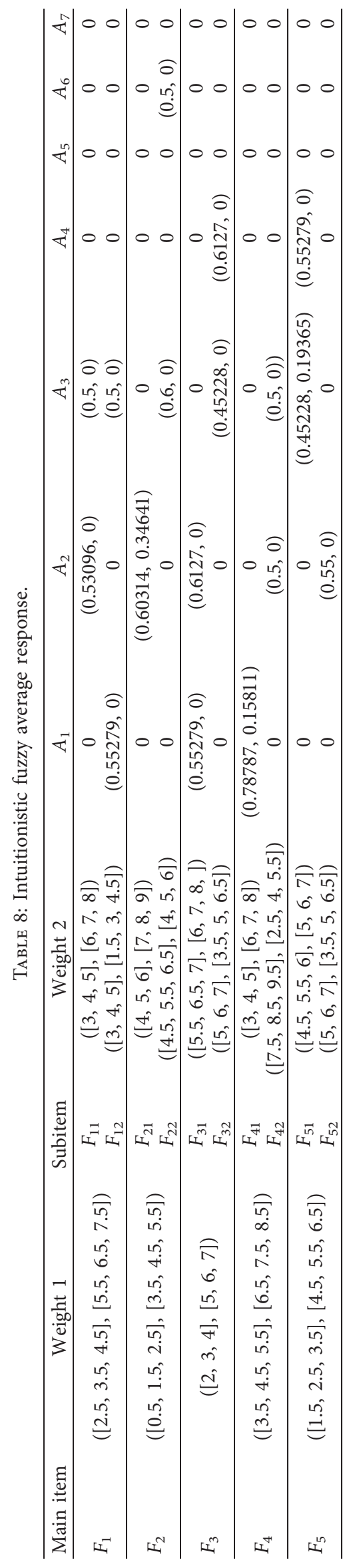


TABLE 9: Intuitionistic fuzzy aggregative investment rate at different hesitancy levels.

\begin{tabular}{lr}
\hline Hesitancy level & Investment benefit \\
\hline 0.025 & $(0.491689,0.01748)$ \\
0.05 & $(0.452331,0.021309)$ \\
0.075 & $(0.431945,0.027895)$ \\
0.1 & $(0.416548,00.028696)$ \\
\hline
\end{tabular}

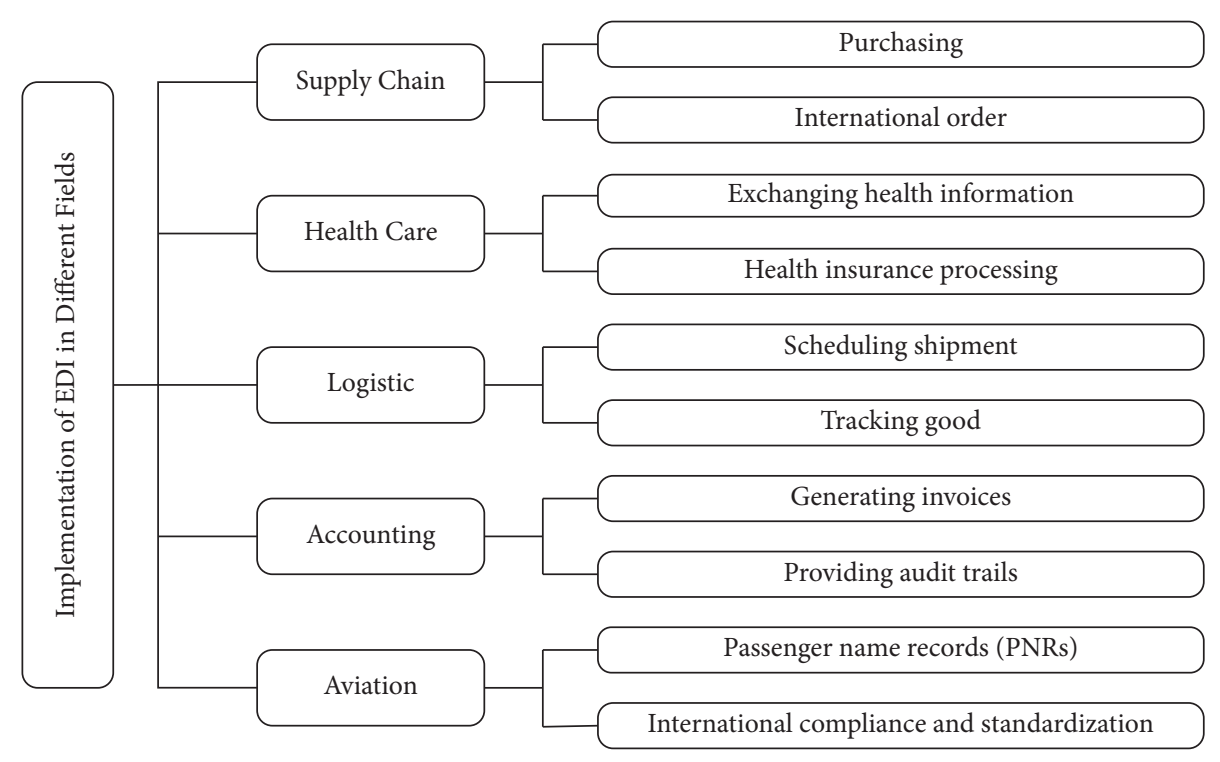

FIgURE 1: hierarchical model on the implementation of EDI in Pakistan with respect to its use in various fields.

Step 1: the intuitionistic fuzzy average response of two experts about EDI implementation $R$ is

$R=\left[\begin{array}{ccccccc}(0,0) & (0,0) & (0,0) & (0,0) & (0.6536,0) & (0.7764,0) & (0,0) \\ (0,0) & (0,0) & (0,0) & (0.8470,0.1276) & (0,0) & (0,0) & (0,0) \\ (0,0) & (0,0) & (0,0) & (0.2918,0) & (0.4523,0) & (0,0) & (0,0) \\ (0,0) & (0,0) & (0.7265,0.2478) & (0,0) & (0,0) & (0,0) & (0,0) \\ (0,0) & (0.8375,0.1361) & (0,0) & (0,0) & (0,0) & (0,0) & (0,0) \\ (0,0) & (0,0) & (0,0) & (0,0) & (0,0) & (0.8419,0.1436) & (0,0) \\ (0.9252,0.0367) & (0,0) & (0,0) & (0,0) & (0,0) & (0,0) & (0,0) \\ 0 & (0,0) & (0,0) & (0,0) & (0,0) & (0,0) & (0.8604,0.1146) \\ (0.9032,0.0707) & 0 & (0,0) & (0,0) & (0,0) & (0,0) & (0,0)\end{array}\right]$


Step 2: the first stage intuitionistic fuzzy aggregative assessment for different fields $C_{i}$ is as

$$
R^{*}=\left[\begin{array}{ccc}
(0,0) & (0,0) & (0,0) \\
(0,0) & (0,0) & (0.2787,0.0951) \\
(0,0) & (0.2483,0.0394) & (0,0) \\
(0.1535,0.0061) & (0,0) & (0,0) \\
(0.9168,0.0573) & (0,0) & (0,0)
\end{array}\right.
$$

Step 3: the main intuitionistic fuzzy aggregative assessment membership and nonmembership rate for all linguistic variables $A_{1}, A_{2}, A_{3}, A_{4}, A_{5}, A_{6}, A_{7}$ are

$$
\begin{aligned}
& (0.1128,0.0064),(.0441, .0072),(.0695,0.0237) \\
& \quad \cdot(0.1558,0.158),(0,0),(0.2410,0.0185),(0,0.0173) .
\end{aligned}
$$

and this IF EDI implementation rate is represented as

$$
\begin{aligned}
\bar{I}= & \frac{(0.1128,0.0064)}{A_{1}}+\frac{(0.0441,0.0072)}{A_{2}}+\frac{(0.0695,0.0237)}{A_{3}} \\
& +\frac{(0.1558,0.158)}{A_{4}}+\frac{(0,0)}{A_{5}}+\frac{(0.2410,0.0185)}{A_{6}}+\frac{(0,0.0173)}{A_{7}} .
\end{aligned}
$$

Defuzzified value of the above EDI implementation membership and nonmembership rate with 0.025 hesitancy error is $(0.3440,0.0472)$. The intuitionistic fuzzy EDI implementation rate is 0.3440 , approximately 34 percent. The Express Tribune [18] published report in 2021 newspaper regarding the EDI implementation rate. Pakistan's domestic industry is destroying massive under-invoicing, especially by commercial importers and suggested in report that "the government of Pakistan must insist on Electronic Data Interchange (EDI), for both FTA and non-FTA imports from major trading partners, and in future, the requirement of EDI should be made compulsory in important fields of country. The less use of EDI in Pakistan is verified by intuitionistic fuzzy EDI implementation rate which shows the significance of proposed methodology. This methodology is novel as the aggregative assessment rate is developed with the hesitancy level.

\section{Conclusion}

Aggregative assessment analysis of linguistic variables obtained from survey sampling may not be reliable due to respondents' ambiguity in human minds. Fuzzy assessment methods are developed to find the benefit rate to select the best facility site selection via survey sampling to tackle the problem of

$\left.\begin{array}{cccc}(0.3566, .0537) & (0.3784,0) & (0.4495,0) & (0,0) \\ (0.2029,0) & (0.2788,0) & (0,0) & (0,0) \\ (0,0) & (0,0) & (0.5978,0.1020) & (0,0) \\ (0,0) & (0,0) & (0,0) & (0.7176,0.0956) \\ (0,0) & (0,0) & (0,0) & (0,0)\end{array}\right]$.

vagueness but do not represent the hesitancy level on the results. However, the intuitionistic fuzzy aggregative benefit rate in survey sampling analysis with the hesitancy level close to zero gives better aggregative evaluation. The results at different hesitancy levels showed that, by increasing hesitancy level, the intuitionistic Fuzzy aggregative membership benefit rate decreases and nonmembership rate increases. Thus, in survey sampling, usually, the aggregative assessment of linguistic variables in IF sense provides better results than fuzzy aggregative assessment by providing the hesitancy level of responses. Real-life application of the IF EDI implementation rate in Pakistan with the hesitancy level showed that instead of gathering information from the large sample who have less information about linguistic terms, the IF assessment rate from experts' opinion provides the results not only with the hesitancy level but also with a significant reduction in time, effort, and especially in cost. Usually, recent research studies in survey sampling contain imprecise data, the analysis of such data can obtain more reliability by applying intuitionistic fuzzy set theory, which implies the hesitancy level on obtained data. The intuitionistic fuzzy rate can be applied to all type of surveys from any field, where data are obtained via questionnaire containing linguistic variables.

\section{Data Availability}

No data were used to support this study.

\section{Disclosure}

The statements made and views expressed are solely the responsibility of the author.

\section{Conflicts of Interest}

The authors declare that they have no conflicts of interest.

\section{Authors' Contributions}

All authors contributed equally and significantly in writing this article. All authors read and approved the final manuscript. 


\section{Acknowledgments}

The sixth author (YUG) would like to acknowledge that this publication was made possible by a grant from Carnegie Corporation of New York.

\section{References}

[1] M. H. Khani and A. Afkhami, The Study of Sociolinguistic Patterns of Turkish Dialect of Qazvin: A Survey of the Effects of Social Variables on Phonological Changes, 2020.

[2] L. A. Zadeh, "Fuzzy sets," Information and Control, vol. 8, no. 3, pp. 338-353, 1965.

[3] L. A. Zadeh, "The concept of a linguistic variable and its application to approximate reasoning-II," Information Sciences, vol. 8, no. 4, pp. 301-357, 1975b.

[4] L. A. Zadeh, "The concept of a linguistic variable and its application to approximate reasoning-I," Information Sciences, vol. 8, no. 3, pp. 199-249, 1975a.

[5] L. Lin and H.-M. Lee, "Fuzzy assessment method on sampling survey analysis," Expert Systems with Applications, vol. 36, no. 3, pp. 5955-5961, 2009.

[6] L. Lin and H. M. Lee, "A new assessment model for global facility site selection," International Journal of Innovative Computing Information and Control, vol. 5, 2008.

[7] C. Li, K. K. Chang, and S. M. Ou, "Using fuzzy sampling survey to explore the factors influencing regional cooperation Changsha-Zhuzhou-Xiangtan case," IOP Conference Series: Materials Science and Engineering, vol. 1113, no. 1, Article ID 012020, 2021.

[8] F. F. Tavares and G. Betti, "The pandemic of poverty, vulnerability, and COVID-19: evidence from a fuzzy multidimensional analysis of deprivations in Brazil," World Development, vol. 139, Article ID 105307, 2021.

[9] K. T. Attanasov, "New operations defined over the intuitionistic fuzzy sets," Fuzzy Sets and Systems, vol. 61, no. 2, pp. 137-142, 1994.

[10] K. T. Attanasov, "More on intuitionistic fuzzy sets," Fuzzy Sets and Systems, vol. 33, no. 1, pp. 37-45, 1989.

[11] K. T. Attanasov, "Intuitionistic fuzzy sets," Fuzzy Sets and Systems, vol. 20, no. 1, pp. 87-96, 1986.

[12] C. Radhika and R. Parvathi, "Defuzzification of intuitionistic fuzzy sets," Notes Intuitionistic Fuzzy Sets, vol. 22, no. 5, pp. 19-26, 2016.

[13] K. Thangavelu, G. Uthra, and B. Amutha, "A new defuzzyfication measure for solving intuitionistic fuzzy assessment problem," International Journal of Pure and Applied Mathematics, vol. 106, no. 7, pp. 69-76, 2016.

[14] G. Işık and İ. Kaya, "Design and analysis of acceptance sampling plans based on intuitionistic fuzzy linguistic terms," Iranian Journal of Fuzzy Systems, vol. 18, no. 6, pp. 101-118, 2021.

[15] U. L. Yuhana, N. Z. Fanani, E. M. Yuniarno, S. Rochimah, L. T. Koczy, and M. H. Purnomo, "Combining fuzzy signature and rough sets approach for predicting the minimum passing level of competency achievement," International Journal of Artificial Intelligence, vol. 18, pp. 237-249, 2020.

[16] R.-E. Precup, T.-A. Teban, A. Albu, A.-B. Borlea, I. A. Zamfirache, and E. M. Petriu, "Evolving fuzzy models for prosthetic hand myoelectric-based control," IEEE Transactions on Instrumentation and Measurement, vol. 69, no. 7, pp. 4625-4636, 2020.

[17] X. Zhang and P. Liu, "Method for aggregating triangular fuzzy intuitionistic fuzzy information and its application to decision making/numanomụ neapibrèžtụjụ aibiụ teorija Ir jos taikymas priimant sprendimus," Technological and Economic Development of Economy, vol. 16, no. 2, pp. 280-290, 2010.

[18] S. Siddiqui, PBC Proposes Pro-growth Budget for FY22, https://tribune.com.pk, 2021. 